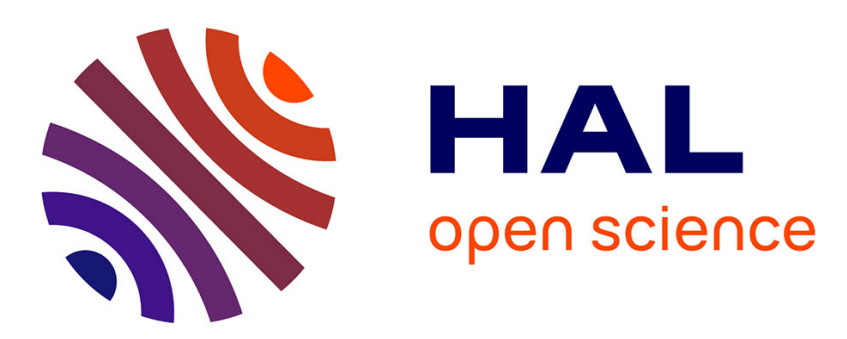

\title{
Absorption ultrasonore et célérité de groupe: analyse temps-fréquence d'un mécanisme de relaxation
}

\author{
J. Guilbot
}

\section{To cite this version:}

J. Guilbot. Absorption ultrasonore et célérité de groupe: analyse temps-fréquence d'un mécanisme de relaxation. Journal de Physique IV Proceedings, 1994, 04 (C5), pp.C5-721-C5-724. 10.1051/jp4:19945155 . jpa-00252834

\section{HAL Id: jpa-00252834 https://hal.science/jpa-00252834}

Submitted on 1 Jan 1994

HAL is a multi-disciplinary open access archive for the deposit and dissemination of scientific research documents, whether they are published or not. The documents may come from teaching and research institutions in France or abroad, or from public or private research centers.
L'archive ouverte pluridisciplinaire HAL, est destinée au dépôt et à la diffusion de documents scientifiques de niveau recherche, publiés ou non, émanant des établissements d'enseignement et de recherche français ou étrangers, des laboratoires publics ou privés. 


\title{
Absorption ultrasonore et célérité de groupe : analyse temps-fréquence d'un mécanisme de relaxation
}

\section{J. GUILBOT}

\author{
Laboratoire d'Acoustique, Systèmes, Signaux et SOnar (LASSSO), ICPI Lyon, 25 rue du Plat, \\ 69288 Lyon cedex 02, France
}

\begin{abstract}
résumé: Dans un premier temps, nous rappelons l'expression du coefficient d'absorption ultrasonore lié à un mécanisme de relaxation. Un tel mécanisme entraîne une dispersion de célérité de phase des ondes ultrasonores lors de leur propagation. Une expression de cette célérité peut être obtenue à partir des relations de Kramers-Kronig. Dans un deuxième temps, nous montrons que l'étude de la dispersion de célérité de groupe associée, fournit une méthode pertinente d'estimation des paramètres caractéristiques du phénomène étudié. La simulation de la propagation d'ondes ultrasonores dans un tel milieu illustre, à partir d'une analyse temps-fréquence des signaux dispersifs, l'intérêt apporté par cette approche.
\end{abstract}

abstract: We first remind the expression of the ultrasonic absorption coefficient associated to a relaxation mechanism. Such a phenomenon leads to a phase velocity dispersion of ultrasonic waves which propagate in the medium. The explicit formulation of the phase velocity can be obtained from the Kramers-Kronig relations. We show that the study of the group velocity dispersion provides a relevant approach to estimate the relaxation parameters. Simulating the propagation of ultrasonic waves in such media illustrates the efficiency of a timefrequency analysis for dispersive signals.

\section{INTRODUCTION.}

L'étude de l'absorption ultrasonore dans les milieux fluides suscite depuis longtemps l'attrait de nombreux travaux de recherche. A côté de l'absorption dite classique, due aux pertes par viscosité et par conduction thermique, on distingue un amortissement lié à un processus intermoléculaire appelé phénomène de relaxation. Ce phénomène particulier s'accompagne d'une dispersion de célérité des ondes ultrasonores lors de leur propagation dans le fluide. L'étude conjointe de l'absorption et de la dispersion constitue un moyen efficace d'investigation des propriétés physiques du milieu et plus particulièrement de ses propriétés intermoléculaires. Les études menées jusqu'à présent se sont limitées aux relations liant absorption et dispersion de célérité de phase ${ }^{1,2}$. Nous montrons dans cet article que l'étude de la dispersion de célérité de groupe permet, dans le cas d'un mécanisme de relaxation à une seule fréquence de relaxation, d'atteindre directement certains paramètres descriptifs du phénomène.

\section{ABSORPTION ET CELERITE DE PHASE.}

Nous nous limiterons par la suite au cas d'un milieu fluide présentant une seule fréquence de relaxation (fluide "idéal") bien que la majorité des fluides réels présentent une distribution de temps de relaxation ${ }^{3}$. Lorsque l'absorption de l'énergie acoustique ultrasonore résulte d'un mécanisme de relaxation, le coefficient d'absorption $\alpha(v)$ ( $v$ fréquence) est donné par la relation: 


$$
\frac{\alpha(v)}{v^{2}}=B+\frac{A}{\beta(v)} \quad \text { avec } \quad \beta(v)=1+\left(\frac{v}{v_{0}}\right)^{2}
$$

où $v_{0}$ est la fréquence de relaxation et où $\mathrm{A}$ et $\mathrm{B}$ sont des constantes caractéristiques du fluide.

La figure 1 présente les variations du coefficient $\alpha(v) / v^{2}$ dans le cas d'une solution d'acide éthanoïque 4 à la température $\theta=17^{\circ} \mathrm{C}\left(\mathrm{B}=1,6.10^{-13} \mathrm{~s}^{2} / \mathrm{m}, \mathrm{A}=1,78.10^{-10} \mathrm{~s} / \mathrm{m}, \mathrm{v}_{\mathrm{o}}=488 \mathrm{kHz}\right)$.

Un mécanisme de relaxation entraîne dans un tel milieu une dispersion de célérité de phase des ondes ultrasonores. Plusieurs auteurs se sont déjà intéressés à cette correspondance, en soulignant l'apport des relations de Kramers-Kronig dans cette étude ${ }^{5,6}$. L'application de ces relations à partir de l'expression (1) de $\alpha(v)$ permet d'obtenir une formulation de la célérité de phase $c_{\varphi}(v)$ :

$$
c_{\varphi}(v)=\frac{c_{\infty}}{1+\frac{\gamma}{\beta(v)}} \quad \text { avec } \quad \gamma=\frac{A v_{0} c_{\infty}}{2 \pi}
$$

où $\mathrm{c}_{\infty}$, fonction des paramètres $\mathrm{A}, \mathrm{B}$ et $\mathrm{v}_{\mathrm{o}}$, est la limite asymptotique haute fréquence de la célérité de phase.

\section{DISPERSION DE CELERITE DE GROUPE.}

Afin de parfaire la caractérisation acoustique du milieu considéré, il nous est apparu intéressant d'étudier les variations de la célérité de groupe $c_{\mathrm{g}}(v)$. D'un point de vue expérimental, cette démarche se justifie d'autant plus, que l'utilisation d'outil d'analyse temps-fréquence, tel que la distribution de WignerVille$^{7}$, permet un accès aisé à la loi đe dispersion de célérité de groupe de signaux large bande. La relation liant la célérité de groupe à la célérité de phase est rappelée par l'expression (3)

$$
c_{g}(v)=c_{\varphi}(v) /\left(1-\frac{v}{c_{\varphi}(v)} \frac{d c_{\varphi}(v)}{d v}\right)
$$

En reprenant l'expression (2) de $c_{\varphi}(v)$, le calcul du second membre de (3) conduit à $c_{g}(v)$ :

$$
c_{g}(v)=c_{\varphi}(v)\left(1+\frac{2\left(v / v_{0}\right)^{2}}{\beta(v)(1+(\beta(v) / \gamma))-2\left(v / v_{0}\right)^{2}}\right)
$$

L'étude du maximum de la fonction $c_{g}(v)$ fournit la relation remarquable suivante:

$$
\frac{\partial c_{\mathrm{g}}(v)}{\partial v}=0 \quad \Leftrightarrow \quad v=\sqrt{3} v_{\mathrm{o}}
$$

On trouve là une méthode pertinente d'estimation du paramètre caractéristique $v_{0}$. D'autres propriétés non moins intéressantes de la célérité de groupe sont à souligner. 
La limite asymptotique haute fréquence est égale à $c_{\infty}$. A la fréquence particulière $v_{0}$, la célérité de groupe est égale à $\mathrm{c}_{\infty}$. Cette dernière propriété peut être mise à profit pour mesurer ce paramètre. La valeur $c_{\mathrm{gm}} \mathrm{du}$ maximum de la célérité de groupe est donnée par l'expression:

$$
c_{g m}=\frac{8 c_{\infty}}{8-\gamma}
$$

ce qui permet d'atteindre le second paramètre caractéristique $\mathrm{A}$ ( $\mathrm{c}_{\infty}$ étant alors connu):

$$
A=\frac{16 \pi}{v_{0} c_{\infty}}\left(1-\frac{c_{\infty}}{c_{g m}}\right)
$$

La figure 2 présente la courbe de dispersion de célérité de groupe pour la solution d'acide éthanoïque précédente $\left(c_{\infty}=1600 \mathrm{~m} / \mathrm{s}\right)$.

Dans le cadre général d'une étude expérimentale de l'absorption, il peut être intéressant de passer de la relation de dispersion de célérité de groupe à celle de la célérité de phase. Ce passage est possible à partir de l'expression $(5)^{8}$ :

$$
\mathrm{c}_{\varphi}(v)=v / \int_{0}^{\nu} \frac{1}{c_{g}(\xi)} d \xi
$$

La figure 2 présente la courbe de dispersion de célérité de phase obtenue après intégration de l'inverse de la fonction $c_{\mathrm{g}}(\mathrm{v})$ (où $\mathrm{c}_{\mathrm{g}}(\mathrm{v})$ est donné par (4)).

\section{SIMULATIONS.}

Nous présentons les résultats issus de la simulation numérique de la propagation d'ondes ultrasonores dans une solution d'acide éthanoïque ${ }^{4}$. Les signaux sont synthétisés par transformation de Fourier inverse, la loi de dispersion de célérité de phase étant connue. Le spectre du signal émis est décrit par une gaussiène de fréquence centrale $800 \mathrm{kHz}$ et de bande fréquentielle 1 octave a $-6 \mathrm{~dB}$. Les signaux sont bruités (bruit blanc additif), le rapport signal sur bruit étant égal à $40 \mathrm{~dB}$. Le calcul de la distribution Pseudo Wigner-Ville Lissée $e^{7}$ de deux signaux (figures 3 et 4), pour deux distances de propagation différentes, permet le calcul du retard de groupe associé à chacun des signaux puis l'estimation des variations de la célérité de groupe ${ }^{9}$ (figure 2).

Bien que la dispersion de célérité de groupe soit faible (inférieure à $3 \%$ ), l'analyse temps-fréquence des signaux dispersifs permet de retrouver la loi de dispersion de célérité de groupe (écart entre les courbes théorique et estimée inférieur à $0,1 \%$ ). La fréquence de relaxation, la limite asymptotique haute fréquence ainsi que le paramètre A peuvent alors être estimés à partir des propriétés ennoncées.

\section{CONCLUSION.}

Nous avons souligné, dans le cas d'un mécanisme de relaxation, l'intérêt apporté par l'étude de la dispersion de célérité de groupe sur la caractérisation de l'absorption ultrasonore. La valeur maximale de la célérité de groupe est obtenue pour une fréquence égale à $\sqrt{3}$ fois la fréquence de relaxation, valeur permettant par ailleurs une estimation du paramètre caractéristique $A$. Une validation expérimentale, à partir d'une analyse temps-fréquence des signaux dispersifs, devra compléter ces premiers résultats. L'extension de cette étude, au cas de milieux présentant plusieurs fréquences de relaxation, pourra alors être étudiée. 


\section{Références}

[1] Gasse S. et Emery J., Acustica 68 (1989) 142-149.

[2] Makarewicz R., Acustica 67 (1988) 45-51.

[3] Narasimham A.V. and Seshadri A.T., Acustica 71 (1990) 223-229.

[4] Bhatia A.B., Ultrasonic absorption (Dover Publications, New York, 1985) pp. 194-263.

[5] Gasse S., Emery J. et Zarembowitch A., Revue d'Acoustique 16 (1971) 263-268.

[6] Angel Y.C. and Achenbach J.D., J. Acoust. Soc. Am. 90 (1991) 2757-2762.

[7] Flandrin P. et Escudié B., Traitement du Signal 2 (1985) 143-150.

[8] Guilbot J., J. Acoustique 5 (1992) 635-638.

[9] Sessarego J.P. et al., J. Acoustique 3 (1990) 273-280.

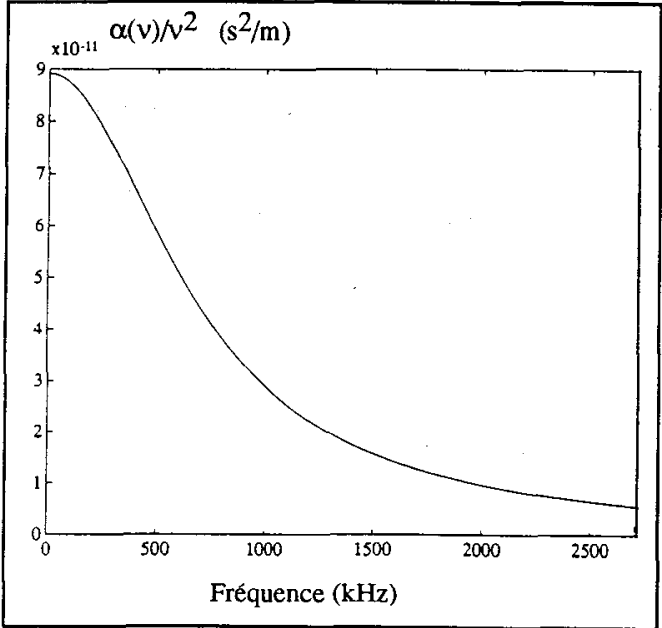

Figure 1: Coefficient d'absorption ultrasonore: cas d'un mécanisme de relaxation.

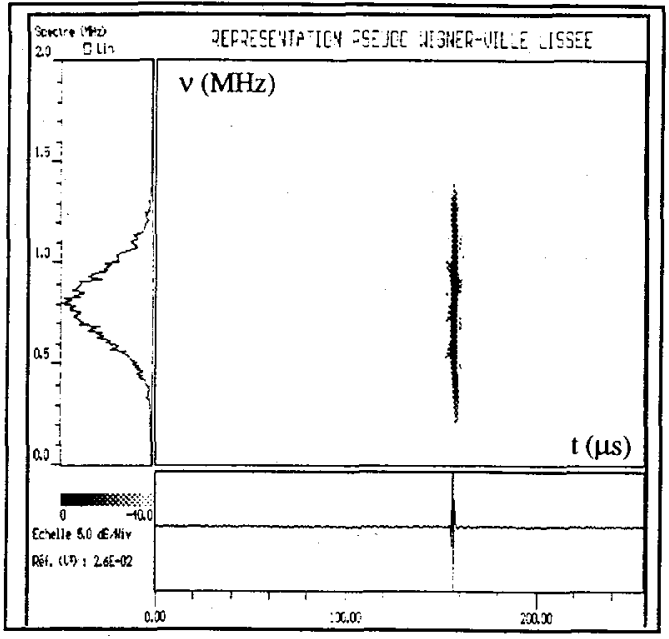

Figure 3: Représentation temps-fréquence du signal reçu après $250 \mathrm{~mm}$ de propagation.

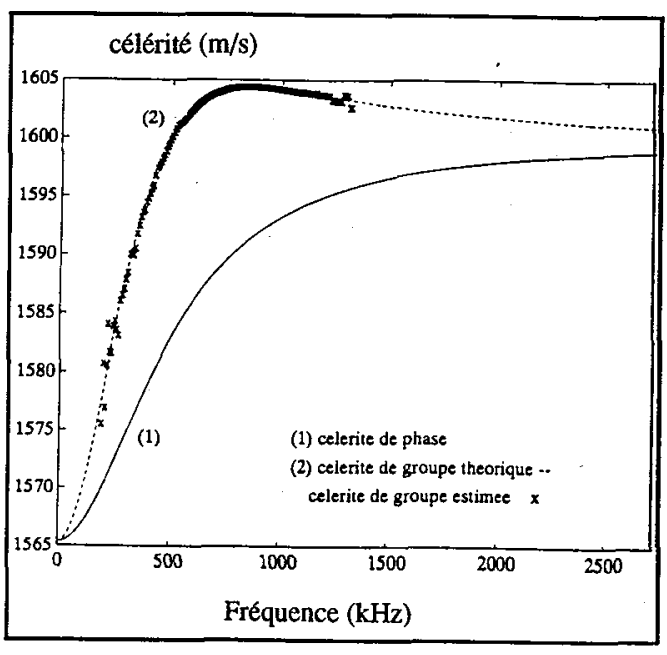

Figure 2: Courbes de dispersion de célérité de phase et de groupe.

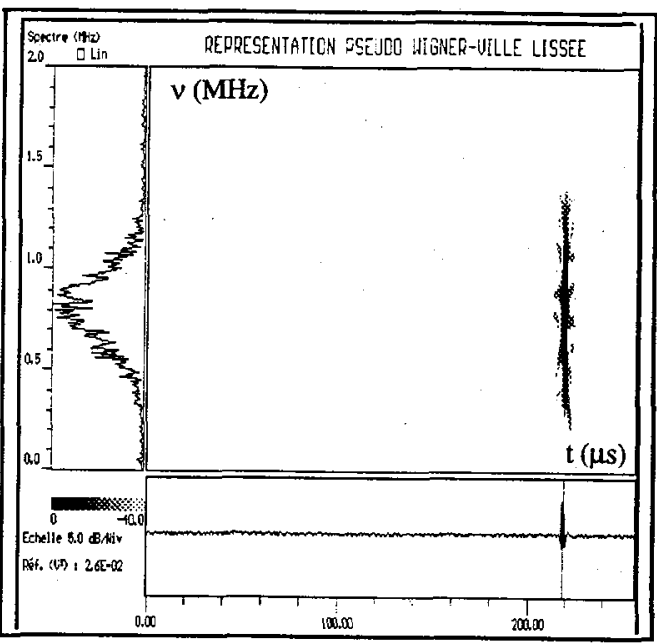

Figure 4: Représentation temps-fréquence du signal reçu après $350 \mathrm{~mm}$ de propagation. 